\title{
A Novel Scheme For Dynamically Tracking Solar Panel
}

\author{
Sali Naveen ${ }^{1}$, M. Ahmed Ali Baig ${ }^{2}$ And Ashutosh Saxena ${ }^{3}$ \\ ${ }^{1}$ P.G Scholar, Department Of Mechanical, Cmr Technical Campus/Jntuh, India \\ ${ }^{2}$ Professor, Department Of Mechanical, Cmr Technical Campus / Jntuh, India \\ ${ }^{3}$ Professor, Department Of Cse, Cmr Technical Campus / Jntuh, India
}

\begin{abstract}
Usage of electricity is increasing day-by-day, with limited fossil fuel. Researchers always like to explore other sources. Solar energy is one such source. Where in the sunlight is captured via solar cells which then transform heat energy to electrical energy to be store for consumption. With the rotation of earth, the direction of sun light changes throughout the day. Attempts are being made to rotate the solar panel by various means to capture maximum sunlight. In this paper we attempt to review the major work carried out in solar tracking system and also propose a novel method for tracking using GPS based infrastructure
\end{abstract}

Keywords : Solar tracker, solar panel, solar power, photovoltaic cell.

\section{Introduction}

Today the usage of electricity in the universe is gradually increasing day-by- day. To overcome the lag of electricity, there are different types of techniques available. One of them is to utilize the solar energy to generate electricity directly from the sunlight by photovoltaic conversion. Solar energy has long been regarded as an ideal energy source but for the fact that we knew little to tap or use it to our advantage. The advancement in science and technology brought out by mankind had lead to developments like the photovoltaic cell (PV cells). Solar panels comprise of a number of such PV Cells. The output of the Solar Panel is proportional to the intensity of incident radiation from the sun.

A field of young sunflowers will slowly rotate from east to west over the course of a sunny day, each leaf seeking out as much sunlight as possible as the sun moves across the sky through an adaptation called heliotropism. It's a clever bit of natural engineering. On similar line a solar tracking system is designed for power generation from sunlight. This method of power generation is simple and is taken from natural resource for maximum sunlight to generate power. This project helps for power generation by setting the equipment to get maximum sunlight automatically. Efforts are there to automate the tracking system for maximum intensity of light. When there is decrease in intensity of light, this system automatically changes its direction to get maximum intensity of light.

In this paper we attempt to review the works carried out in this area and propose a novel method for Automatic solar tracking using GPS. The organization of the paper is follows: Section 2: presents several Methods to Drive Solar Trackers, in Section 3: Types of Tracking Systems and Factors Affecting Tracing Efficiency are discussed, in Section 4: we cover Solar Tracking Applications along with few advantages and disadvantages, in Section 5: Review of Solar Tracking System is presented along with the brief of our scheme this is followed by Conclusion and later the references.

\section{Methods To Drive Solar Trackers}

\subsection{Active tracker}

Active trackers make use of motors and gear trains for direction of the tracker as commanded by the controller responding to the solar direction. The position of the sun is monitored throughout the day. When the tracker is subjected to darkness, it either sleeps or stops depending on the design. This is done using sensors that are sensitive to light such as Lower Density Radiations (LDR). Their voltage output is put into a microcontroller that then drives actuators to adjust the position of the solar panel.

\subsection{Passive solar tracking}

Passive trackers use a low boiling point compressed gas fluid driven to one side or the other to cause the tracker to move in response to an imbalance. Because it is a non precision orientation it is not suitable for some types of concentrating photovoltaic collectors but works just fine for common PV panel types. These have viscous dampers that prevent excessive motion in response to gusts of wind. 


\subsection{Chronological solar tracking}

A chronological tracker counteracts the rotation of the earth by turning at the same speed as the earth relative to the sun around an axis that is parallel to the earth. To achieve this, a simple rotation mechanism is devised which enables the system to rotate throughout the day in a predefined manner without considering whether the sun is there or not. The system turns at a constant speed of one revolution per day or 15 degrees per hour. Chronological trackers are very simple but potentially very accurate.

\subsection{Manual tracking}

In some developing nations, drives have been replaced by operators who adjust the trackers. This has the benefits of robustness, having staff available for maintenance and creating employment for the population in the vicinity of the site.

\section{Types of Solar Tracking System}

Usually there are two basic types of solar tracker systems, namely Single axis and Dual axis Trackers.

\subsection{Single axis trackers}

Single axis trackers have one degree of freedom that act as the axis of rotation. The axis of rotation of single axis trackers is aligned along the meridian of the true North. With advanced tracking algorithms, it is possible to align them in any cardinal direction.

Common implementations of single axis trackers include

1. Horizontal single axis trackers (HSAT),

2. Horizontal single axis tracker with tilted modules (HTSAT),

3. Vertical single axis trackers (VSAT),

4. Tilted single axis trackers (TSAT) and

5. Polar aligned single axis trackers (PSAT)
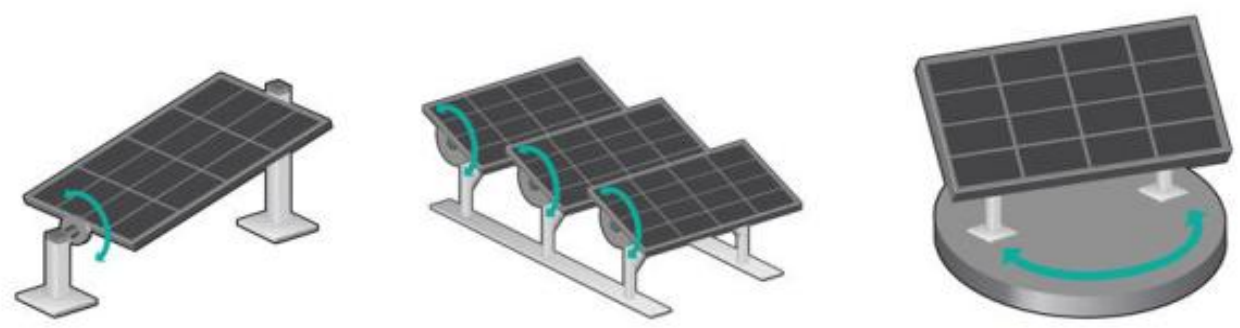

\section{A. Single-axis tracking on a tilted axis B. Single - axis tracking on a horizontal axis C. Single-axis tracking on a vertical axis}

\subsection{Dual axis trackers}

Dual axis trackers have two degrees of freedom that act as axes of rotation. These axes are typically normal to each other. The primary axis is the one that is fixed with respect to the ground. The secondary axis is the one referenced to the primary axis. There are various common implementations of dual trackers. Their classification is based on orientation of their primary axes with respect to the ground.

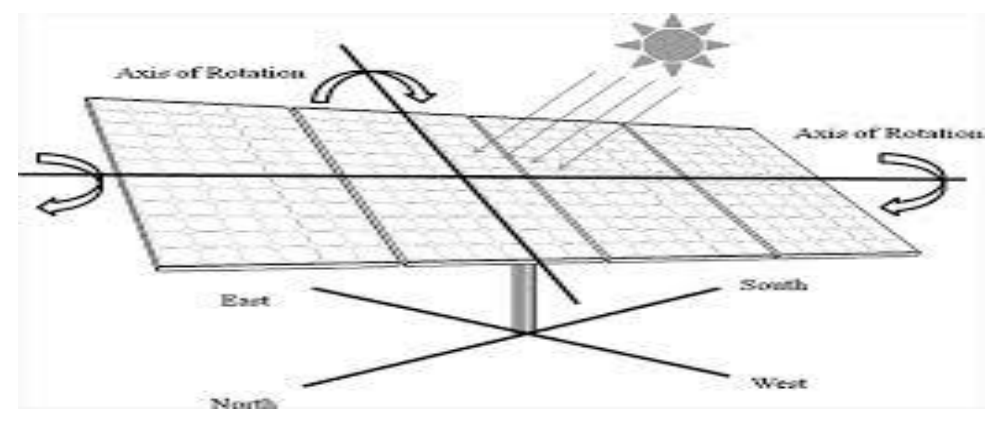

D. Dual Axis Solar Tracker Combines Two Motions while Tracking 


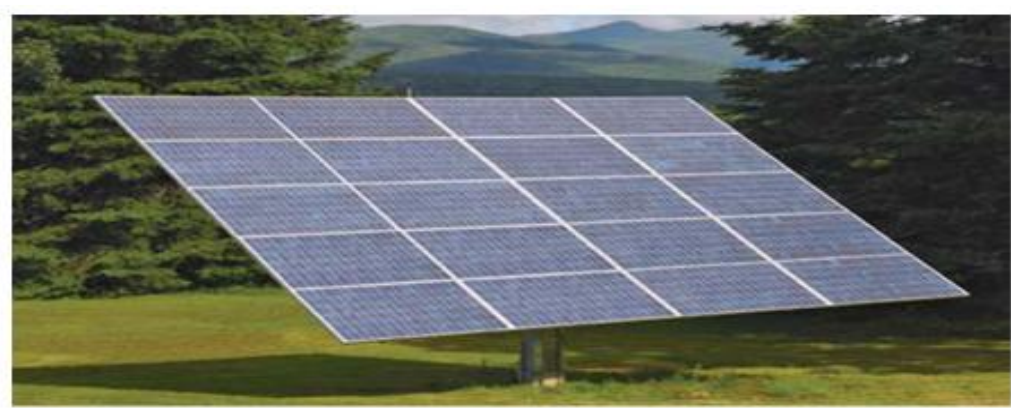

E. Basic model of dual axis tracking system

\subsection{Factors That Affect Solar Tracker Efficiency}

1. Panel Orientation: Solar panel orientation depend upon Azimuth and Zenith angels

Azimuth - This is the compass angle of the sun as it moves through the sky from East to West over the course of the day. Generally, azimuth is calculated as an angle from true south. True south is the direction towards the southern end of the axis about which the earth rotates. The wrong azimuth angle could reduce the energy output of a solar PV array down to $35 \%$.

Zenith - This is the angle of the sun looking up from ground level or the horizon. The zenith angle of the sun varies throughout the day in the form of an arc with the sun reaching its maximum elevation (also called solar altitude) around midday.

2. Roof and Panel Pitch: The "pitch" or tilt of roof can affect the number of hours of sunlight you receive on an average day throughout the year. Large commercial systems have solar tracking systems that automatically follow the sun's tilt through the day.

3. Temperature: Some panels like it hot but most don't. So, panels typically need to be installed a few inches above the roof with enough air flow to cool them down. Some photovoltaic panels are designed to be more efficient in hotter climates.

4. Shade: Basically, shade is the enemy of solar power. With poor solar design, even a little shade on one panel can shut down energy production on all of your other panels (like a bad bulb in a string of series-lights).

\section{Solar Tracking Application}

Solar trackers are devices used to orient photovoltaic panels, reflectors, lenses or other optical devices toward the sun. Since the sun's position in the sky changes with the seasons and the time of day, trackers are used to align the collection system to maximize energy production.

Several factors must be considered when determining the use of trackers. Some of these include: the solar technology being used, the amount of direct solar irradiation, feed-in tariffs in the region where the system is deployed, and the cost to install and maintain the trackers.

\subsection{Advantages}

- Trackers generate more electricity than their stationary counterparts due to increased direct exposure to solar rays. This increase can be as much as 10 to $25 \%$ depending on the geographic location of the tracking system.

- There are many different kinds of solar trackers, such as single-axis and dual-axis trackers, all of which can be the perfect fit for a unique jobsite. Installation size, local weather, degree of latitude and electrical requirements are all important considerations that can influence the type of solar tracker best suited for a specific solar installation.

- Solar trackers generate more electricity in roughly the same amount of space needed for fixed tilt systems, making them ideal for optimizing land usage.

- In some places utilities offer Time of Use (TOU) rate plans for solar power, which means the utility will purchase the power generated during the peak time of the day at a higher rate. In this case, it is beneficial to generate a greater amount of electricity during these peak times of day. Using a tracking system helps maximize the energy gains during these peak time periods.

- Advancements in technology and reliability in electronics and mechanics have drastically reduced longterm maintenance concerns for tracking systems. 


\subsection{Disadvantages}

- Solar trackers are slightly more expensive than their stationary counterparts, due to the more complex technology and moving parts necessary for their operation. This is usually around a $\$ 0.08-\$ 0.10 / \mathrm{W}$ increase depending on the size and location of the deployment.

- Even with the advancements in reliability there is generally more maintenance required than a traditional fixed rack, though the quality of the solar tracker can play a role in how much and how often this maintenance is needed.

- Trackers are a more complex system than fixed racking. This means that typically more site preparation is needed, including additional trenching for wiring and some additional grading.

- Solar trackers are generally designed for climates with little to no snow making them a more viable solution in warmer climates. Fixed racking accommodates harsher environmental conditions more easily than tracking systems.

- Fixed racking systems offer more field adjustability than single-axis tracking systems. Fixed systems can generally accommodate up to $20 \%$ slopes in the E/W direction while tracking systems typically offer less of a slope accommodation usually around $10 \%$ in the N/S direction.

\section{Review of solar tracking system}

In 1975, one of the first automatic solar tracking systems was presented by McFee, in which an algorithm was developed to compute total received power and flux density distribution in a central receiver solar power system. By subdividing each mirror into 484 elements and summing the contributions of all elements, the sun position could be determined with a tracking error tolerance of $0.5^{\circ}-1^{\circ}$. Several years later, Semma and Imamru used a simple microprocessor to adaptively adjust the positions of the solar collectors in a photovoltaic concentrator such that they pointed toward the sun at all times. Mathematical theories of tracking error distributions were also developed to improve the algorithms of determining sun position [1] In 1983, Al-Naima and Yaghobian developed a solar tracking system featuring a two-axis equatorial mount and a microprocessor, in which the tracking operation was performed on the basis of the astronomical coordinates of the sun. The experimental results demonstrated that the proposed system yielded a significantly better tracking performance than that obtained by a conventional sensor- controlled system [2]. Several years later, Lorenz proposed a set of design guidelines for a window glazing which rejected solar radiation during the summer, but accepted it during the winter. The design featured a purely passive control algorithm based on seasonal changes in the incident angle of the solar rays [3]. The study of Ashok Kumar Saxena and V. Dutta had designed a microprocessor based controller for solar tracking in 1990. Controller had the capability of acquiring photovoltaic and meteorological data from a photovoltaic system and controlled the battery /load. These features were useful in autonomous PV systems that were installed for system control as well as monitoring in remote areas. Solar tracking was achieved in both open loop and closed loop modes. The system is very useful because, the controller was totally automatic and did not require any operator interference unless needed. [Ashok Kumar Saxena and V.Dutta, 1990] [4]-[5].

A.Konar and A.K. Mandal had given a microprocessor based automatic position control scheme in 1991. They had designed for controlling the azimuth angle of an optimally tilted photovoltaic flat type solar panel or a cylindrical parabolic reflector to get the illuminating surface appropriately positioned for the collection of maximum solar irradiance. The proposed system resulted in saving of energy .It was designed as a pseudo tracker in which step tracking scheme had been used to keep the motor idle to save energy. Temperature variations in environmental parameters caused by fog, rain, snow. Distance from the location where the solar panel was lcoated, the system did not affect proper direction when the system try to finding sun. This system is very efficient because the solar tracker has an energy saving mode. [A. Konar and A.K Mandal, 1991] [6].

Irradiation is the process by which an object is exposed to radiation. The term irradiation usually excludes the exposure to non-ionizing radiation, such as infrared, visible light, microwaves from cellular phones or electromagnetic waves emitted by radio and TV receivers and power supplies [7]. In 1992, Agarwal presented a two-axis tracking system consisting of worm gear drives and four bar-type kinematic linkages to facilitate the accurate focusing of the reflectors in a solar concentrator system. In the same year, Enslin applied the principles of maximum power point tracking (MPPT) to realize a power electronic converter for transforming the output voltage of a solar panel to the required DC battery bus voltage [8].

The study of Zeroual had designed an automatic sun-tracker system for optimum solar energy collection in the year of 1997. They used electro-optical sensors for sun finding and a microprocessor controller in this system. Moreover, this system allowed using solar energy collectors to follow the sun position for optimum efficiency. The solar tracker system had a modular structure which the system have facilitates its application to different systems without any modifications. The system had been applied to control a water heating solar system for houses in domestic uses. Many parameters had been controlled for system security such 
as temperature, pressure and wind velocity. The solar tracker system had been tested for a long period in variable illumination of the sun. The results are showed that it works with high accuracy. In addition to this, the system is very efficient for our solar tracker with use in heating and cooling systems in the houses. [A.Zeroual et al., 1997] [9]. In 1998, Khalifa and Al-Mutawalli developed a two-axis sun tracking system to enhance the thermal performance of a compound parabolic concentrator. The system was designed to track the sun's position every three to four minutes in the horizontal plane and every four to five minutes in the vertical plane. The tracking system was comprised of two identical sub-systems, one for each axis, with each sub-system consisting of two adjacent photo-transistors separated by a partition of a certain height. In the tracking operation, the difference in the voltage signals of the two photo-transistors was amplified and used as a command signal to drive the collector around the corresponding axis until the voltage difference reduced to zero, indicating that the sun's rays were once again normal to the collector surface [10].

In 2004, Roth et al. designed and constructed a sun tracking system in which a pyrheliometer was used to measure the direct solar radiation. The system was controlled by a closed-loop servo system consisting of a four-quadrant photo detector to sense the sun's position and two small DC motors to drive the instrument platform in such a way that the sun's image remained at the center of the four-quadrant detector at all times [11][12]. In 2004, Abdallah and Nijmeh developed an electro-mechanical, two-axis tracking system in which the motion of the sun tracking surface was controlled by an open-loop control algorithm implemented using a PLC unit. A programmable logic controller, PLC, or programmable controller is a digital computer used for automation of typically industrial electromechanical processes, such as control of machinery on factory assembly lines, amusement rides, or light fixtures. The proposed system incorporated two separate tracking motors, namely one motor to rotate the sun tracking surface about the horizontal north-south axis, i.e. to adjust the slope of the surface and the other to rotate the sun tracking surface about the vertical axis, i.e. to adjust the azimuth angle of the surface [13]. In 2005, Alata et al. designed and simulated three sun tracking systems, namely: (1) one-axis sun tracking with the tilted aperture equal to the latitude angle, (2) Equatorial two-axis sun tracking, and (3) azimuth/elevation sun tracking.

For each tracking system, the modeling and controller design tasks were accomplished using the firstorder Sugeno fuzzy inference system. The first two parts of the fuzzy inference process, fuzzifying the inputs and applying the fuzzy operator, are exactly the same [14]. A typical rule in a Sugeno fuzzy model has the form: If Input $1=x$ and Input $2=y$, then Output is $z=a x+b y+c$; For a zero-order Sugeno model, the output level $\mathrm{z}$ is a constant $(\mathrm{a}=\mathrm{b}=0)$. The output level $\mathrm{z}_{\mathrm{i}}$ of each rule is weighted by the firing strength $\mathrm{w}_{\mathrm{i}}$ of the rule. For example, for an AND rule with Input $1=x$ and Input $2=y$, the firing strength is $\mathrm{W}_{\mathrm{i}}=$ And Method (F1(x), F2(y)), Where F1,2 (.) are the membership functions for Inputs 1 and 2 . The final output of the system is the weighted average of all rule outputs, computed as

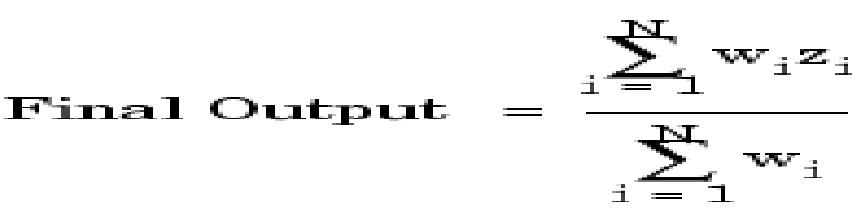

Output level is $\mathrm{z}_{\mathrm{i}}$, and each rule is weighted by the firing strength $\mathrm{w}_{\mathrm{i}}[15]$.

In 2007, Kim presented a robust Maximum Power Point Tracking (MPPT) system based on a sliding mode controller (SMC) for a three-phase grid-connected photovoltaic system. The proposed system comprised both a MPPT controller and a current controller. The MPPT controller generated a current reference directly from the solar array power information, while the current controller used an integral sliding mode scheme to ensure a tight control of the current [16]. In 2007, Chen et al. presented a sun sensor algorithm based on an analogue optical nonlinear compensation measuring principle. In a traditional analogue sun sensor, a thin mask with a square aperture consisting of four slits of equal width is placed above a quadrant detector. The incident sunlight illuminates different positions of the detector depending on its angle relative to the main sensor axis and forms a projective image on the detector's plane [17]-[18]. In 2013 Dante Johnson-Hoyte et al. worked on the use of a highly portable, efficient solar tracker that can be very useful to applications of the military, industrial, or residential variety. To produce an efficient solar generation system solar tracker was designed, built and tested. At most, the solar tracker was perpendicular to the light source within 3 degrees [19].

In 2013Hashem Bukhamsin et al. The difference in power generation of a solar tracker to a static solar panel. The base structure design has been modified for this system. It relies on a free standing, box structure, for easy mobility [20]. In the same year S. Lakeou et al During the course of the year, the array will be tilted around the X-axis progressively from June 21 to December 21 in one direction and from December 22 to June 20 in the 
opposite direction. For a simple tracking system, the daily solar tracking is achieved by rotating the array about the solar tracking axis $\mathrm{Y}$, by equal incremental angular steps $\Delta \phi=150$ [21]. In 2013 Dhanabal.R the LDR with the maximum intensity of the sun's radiation sends stronger signal to the controller which in turn sends signal to the motor to rotate the panel in the direction in which the sun's intensity is Maximum [22]. In 2013 Shrishti Rana A solar tracker is a device for orienting a solar photo voltaic panel, day lighting reflector or concentrating solar reflector or lens toward the sun. Solar power generation works best when pointed directly at the sun, so a solar tracker can increase the effectiveness of such equipment over any fixed position. The solar panels must be perpendicular to the sun's rays for maximum energy generation [23].

Here we propose our scheme which uses GPS based tracking system with step motor. Our system has many advantages over the existing ones as it is place climatic and agnostic. There by it provides the easy installation at any place. Further the step motor also re-aligns the panel for next day automatically and then switches off itself. Also in case of any failure of the system, it automatically recalibrates itself to the position of sun.

\section{Conclusion}

Several solar tracing systems are designed to orient photovoltaic panels, reflectors, lenses and other optical devices towards sun. Since the suns position in the sky changes with seasons, places and the time of the day, trackers are used to align the collection system to maximize energy production in this paper we attempted to review the works conducted y several Engineers \& Scientists and also present the basic idea of our solar tracing system based on GPS. Our scheme is very useful as it is place \& time agnostic. Thus making it more flexible compared to other schemes. Efforts are under way to implement this scheme with a Lab prototype.

\section{References}

[1] McFee R.H. Power collection reduction by mirror surface non flatness and tracking error for a central receiver solar power system. (Appl. Opt. 1975);14:1493-1502

[2] Al-Naima F.M., Yaghobian N.A. Design and construction of a solar tracking system. Solar Wind Technol. 1990; 7:611-617.

[3] Lorenz W. Design guidelines for a glazing with a seasonally dependent solar transmittance. Sol. Energ.1998; 63:79-96.

[4] A.K. Saxena and V. Dutta,(2000) “A versatile microprocessor based controller for solar tracking," in Proc. IEEE, 1990, pp. $1105-1109$.

Ashok Kumar Saxena and V.K Dutta(1990), "A Versatile Microprocessor based Controller for Solar Tracking”, IEEE Conference, Vol. 2, 21 - 25May, 1990, Page(s):1105-1109.

A.Konar and A.K Mandal (1991), "Microprocessor based Sun Tracker", IEEE Proceedings-A, Vol. 138, No.4, July 1991, Page(s):237-241.

[7] https://en.wikipedia.org/wiki/Irradiation.

[8] . Agarwal A.K. Two axis tracking system for solar concentrators. Renew. Energ. 1992; 2:181-182.

[9] A. Zeroual, M. Raoufi , M. Ankrim and A.J. Wilkinson,(1998) "Design and construction of a closed loop Sun Tracker with Microprocessor Management”, International Journal on Solar Energy, Vol. 19, 1998, Page(s): 263-274.

[10] Khalifa A.N., Al-Mutawalli S.S. Effect of two-axis sun tracking on the performance of compound parabolic concentrators. Energ. Convers. Manage. 1998; 39:1073-1079.

[11] Roth P., Georgieg A., Boudinov H. Design and construction of a system for sun-tracking. Renew. Energ.2004; 29:393-402.

[12] Georgiev A., Roth P., Olivares A. Sun following system adjustment at the UTFSM. Energ. Convers. Manage. 2004; 45:1795-1806.

[13] Abdallah S., Nijmeh S. Two axes sun tracking system with PLC control. Energ. Convers. Manage.2004; 45:19311939.

[14] Alata, M.; Al-Nimr, M.A.; Qaroush, Y. Developing a multipurpose sun tracking system using fuzzy control. Energ. Convers. Manage. 2005, 46, 1229-1245.

[15] http://www-rohan.sdsu.edu/doc/matlab/toolbox/fuzzy/fuzzyt27.html.

[16] Kim T.Y., Ahn H.G., Park S.K., Lee Y.K. A novel maximum power point tracking control for photovoltaic power system under rapidly changing solar radiation. Proceedings of ISIE; Pusan, Korea. Jun. 12-16, 2001

[17] Chen F., Feng J., Hong Z. 2006 Digital sun sensor based on the optical vernier measuring principle. Meas. Sci. Technol. 2006; 17:2494-2498.

[18] Chen F., Feng J. Analogue sun sensor based on the optical nonlinear compensation measuring principle. Meas. Sci. Technol. 2007; 18:2111-2115.

[19] Dante Johnson-Hoyte Melanie Li Sing How Dante Rossi Myo Thaw Dual-Axis Solar Tracker [March 10 2013]

[20] Hashem Bukhamsin, Angelo Edge, Roger Guiel, Dan Verne Solar Tracking Structure Design. http://www. Northern Arizona University.com [March 21, 2014]

[21] S. Lakeou E. Ososanya B.O. Latigo W. Mahmoud Design of a Low-cost Solar Tracking Photo-Voltaic (PV) Module and Wind Turbine Combination System. 
[22] Dhanabal.R,Bharathi.V,Ranjitha.R,Ponni.ADeepthi.S,Mageshkannan.P Comparison of Efficiencies of Solar Tracker systems with static panel Single-Axis Tracking System and Dual-Axis Tracking System with Fixed Mount http//www.ijetVol 5 No 2.com [ Apr-May 2013].

[23] Shrishti Rana A Study on automatic dual axis sola tracker system using 555 timer www.ijtra.com Volume 1, [sept 2013]. 